\title{
Emulation of peripherals for automotive electronic control unit
}

\author{
Juraj PANČÍK ${ }^{1}$, Vladimír BENEŠ \\ \{jpancik@bivs.cz ${ }^{1}$, vbenes@bivs.cz ${ }^{2}$ \} \\ Department of Informatics, Vysoká škola regionálního rozvoje a Bankovní institut - AMBIS, a.s., \\ Praha
}

\begin{abstract}
The aim was to develop an embedded system for educational purposes with functions of emulation of some peripherals which are intended for automotive electronic stability control unit (ESC ECU). Emulators of two key ECU peripherals were developed: one for four wheel speed sensors (both two and three current levels types) and second for electronic parking break (EPB) switch based on LIN bus. As real-time processors the Arduino Micro platform was chosen. The up level information system architecture is based on the web server (Raspberry Pi 3 platform) and web browser client and programming was done with JavaScript language for the client (AngularJS framework) and also for server (Node.js).
\end{abstract}

Node.js

Keywords: EPB system; wheel speed sensor; LIN bus; Arduino; Raspberry Pi;

\section{Introduction}

This work presents educational tool for the teaching of modern trends in automotive electronics. The aim was to develop a embedded system with functions of emulation of some peripherals which are intended for automotive electronic control units (ECU, Electronic Control Unit). The ECU is surrounded with peripherals (e.g. by sensor and another hardware parts) and it interacts with their surroundings via car's buses (e.g. CAN, LIN, FlexRAY) or via cable harness and electromechanical systems (e.g. ignition switch or EPB switch). ECU system tests serve to test all required functions ECU. For operations of ECU during system tests is typical that these activities are consistent with the ECU in the car. It is necessary to emulate of ECU's peripherals during systems tests. Therefore emulation of the ECU's peripherals is not only suitable for educational use of the future technicians and engineers but it may also be useful for preparing of ECU system test. In next text is necessary to understand how differences are between simulation and emulation. A simulator is an environment which models but an emulator is one that replicates the usage as on the original device or system. Simulation is when you are replicating, by the means of software, the general behaviour of a system starting from a conceptual model. Emulation is when you are replicating, in a different system, how the original system actually internally works considering each function and their relations. In other words: simulation is based on a software implementation of a model where the internal functions of the original systems are not taken into consideration (for example a "flight simulator" does not have any "component" of an actual aircraft). Emulation is a replica of the internal system functions 
on a different host (for example on a microcontroller platform). Emulators can also be strictly hardware-based and is normally based on a partial or complete "reverse engineering" phase.

\section{ANALYSIS}

\subsection{Description of EPB system}

Since the EPB was first launched in 2001 the number of its functions continues to rise significantly. The EPB offers by far more than the basic apply and release of a conventional parking brake. It interacts with several other driver assistance systems. The driver experiences the EPB system by its functions. He expects safety and reliability at low "costs of ownership" with a highly comfortable "look and feel". The system supplier needs to translate these mainly subjective expectations into physical characteristics of the system and design its components against measurable targets. Figure 2 gives an overview of the EPB system with the functionality perceived by the driver on the one hand and the components with their technical characteristics on the other hand. The EPB system consists of the mechatronic actuators that generate the clamp force necessary to safely hold the vehicle, the conventional calipers that convert clamp force into brake torque, electronic hardware with the Electronic Control Unit (ECU), cable harness and switches and especially the control software providing the functions that the driver will experience. State of the art is to integrate the EPB control unit into one ECU in the car with name electronic stability control system (ESC ECU) [1]. On the market there are Original Equipment Suppliers (OES) - specific solutions as well as OES - independent combinations from different ECU ESC and EPB suppliers. The latter case is commonly called crosswise integration of products from different suppliers and it is dictated and originated in modern global market with automotive components. The integrated EPB system can be divided into two parts: (1) One part of the EPB system contains the parking brake actuator, the parking brake caliper and the actuation logic (Park Brake Control, PBC), (2) The second part of the EPB system, also called the host, contains the EPB power electronics and necessary peripherals and EPB controls the functions as a part of the ESC ECU's embedded software. In crosswise integration projects the OES-EPB is responsible for the first part and the OES-ESC for the second part. The aims of this division are: (a) encapsulation of knowledge about particular components, (b) clearly defined areas of responsibility, (c) independent testing and approval of components from the different suppliers, (d) enabling manufacturer-specific levels of functionality of the individual components. The development and release of such integrated systems needs clear requirements for the interfaces and rules for collaboration between the development partners.

2.2 Peripherals of Electronic Stability Control (ESC ECU) with EPB functionality

The block diagram of the ECU with ESC EPB functionality is showed in Fig.3 [2]. According to it, there are two types peripherals for ESC ECU - peripherals connected to the car's buses (CAN or FlexRAY) and peripherals directly connected to the ECU (wheel speed sensors and the EPB switch). The EPB classical electro-mechanical switch is connected to the ESC ECU with 6 wires (with states: default open, close, Fig.4) or with 8 wires (with AutoHold function). Four pieces of wheel speed sensors (WSS) are connected to the ESC ECU each with two wires and they communicate with ECU by using current signals (the principle of current loop). 


\section{DESIGN AND IMPLEMENTATION}

Wheel speed sensors (WSS) are components with analog current output. WSS are produced as devices with 2 levels (2L) current outputs [3] and with 3 levels (3L) current outputs [4]. Therefore information about wheel position is encoded by two or three levels of current $(7 \mathrm{~mA}$, $14 \mathrm{~mA}$ and $28 \mathrm{~mA}$ current levels). For determine of exact position of the wheel we need to know the wheel circumference and the number of magnets placed around the perimeter of the wheel. LIN (Local Interconnect Network) is a cost-effective and deterministic communication system for connecting ECUs with smart sensors and actuators. The EPB electromechanical switch is in our contribution modified by utilization of the LIN bus, see more in the US patent [5]. Instead normally 6 or 8 wires we use only 3 wires. The trends reducing of number of peripherals wires by utilization of LIN bus can be seen in all modern cars [6]. In our solution of peripherals emulator we tried to use a maximum amount of modern technology well known in the community of developers. We used hardware platforms as Linux based minicomputer Raspberry Pi and as 8 bit AVR microcontroller for real time signal processing - Arduino Micro. The information system architecture is based on the web server and web browser client and programming was done in JavaScript language for the client (AngularJS framework [7]) and also for server (Node.js [8]).

Fig. 8 shows the architecture of the embedded system - right side represents the ECU ("Phantom" ECU side) and it consumes voltage and current signals from emulators at left side. The heart of each emulator's part is Arduino Micro. The programs for all four Arduino's we developed in Arduino IDE environment which is enriched with many libraries and with like C programming language [9]. On the both sides the Arduinos work besides programs in main loop also with programs which were written in interrupt routines. Main loop ensures state machine control and I2C bus communication. Interrupts are derived from serial port (RX or TX) or from internal timers and digital inputs. All distributed Arduino's are controlled via I2C bus from Raspberry Pi [10]. Both Raspberry Pi units have server's role and they have installed Node.js [8]. This server's solution can be programmed with JavaScript language. It means that both sides, clients and servers, can be programmed with common JavaScript language. It includes not only programming of the web server but also the control of $\mathrm{I} 2 \mathrm{C}$ hardware pins for Raspberry $\mathrm{Pi}$ at the I2C master side [11]. There are two client's web sites in this embedded solution. One website serves for setting of parameters for peripheral emulation and another website serves for reading data captured at the ECU side. The developing of both client's web sites we done with utilization JavaScript-based open-source front-end web application framework AngularJS [7] and dynamic JSON data resources which represent inputs / outputs data ARDUINO's.

\section{IMPLEMENTATION}

\subsection{WSS emulator}

We emulate all four car's WSS with 2 or 3 levels current output signal. Computing power of Arduino Micro can serve only for two wheel's pair emulating and it can't independent emulate of each WSS individually. Therefore we can emulate independent front wheels pair as two levels WSS and rear wheels pairs as three levels WSS (or vice versa). Current level pulses were generated via combination of digital controlled analog multiplexer as voltage level selector and open collector driver as voltage - current transformer. Two levels WSS (BOSCH DF11 and 
DF30, (see Fig.5)) can be offered with different signal protocols [3]. The "s" protocol is a square wave signal as rotary speed signal. The " $i$ " protocol is a square wave signal with additional information which is transmitted in the pulse width modulation (PWM) protocol. The width of the square wave impulse includes additional information, while the time between one pulse and the next determines rotary speed information. The " $\mathrm{v"} \mathrm{protocol} \mathrm{is} \mathrm{a} \mathrm{three} \mathrm{level} \mathrm{current} \mathrm{signal}$ that provides wheel speed information and additional information in a serial data protocol in accordance to the "AK-Protocol" [12]. The magnetic sensor on the wheels perimeter generates an output protocol after every detected magnetic signal flank, therefore its output signal frequency is twice as high as for the standard "s" protocol variant. At emulator's ECU side we uses for detection current level pulses from each pair of WSS for ECU dedicated front-end device MAX9921 [13] and ARDUINO Micro (Fig.8) as device for real-time capturing logic level signals from front-end MAX9921. Arduino Micro also ensures I2C communication with up level Raspberry Pi 3 minicomputer. Fig. 6 shows our real measurements of output current for emulation of DF11i in stop vehicle state ("standstill mode").

\section{2... EPB switch emulator}

Implementation of the EPB switch is based on the implementation of one pair of LIN bus nodes. One node is the master (ESC ECU side) and second one is the slave (EPB switch emulator side). The implementation of such pair is not trivial task not only from technical point of view but also from functional safety reasons. EPB switch can play crucial role in car's safety. In order to demonstrate these approaches we added to the standard LIN bus protocol additional safety options. Fig.7 shows the screenshot from LIN bus communication monitor used by us. At this picture are showed LIN bus communication frames recorded between ESC ECU node and EPB switch emulator node. Each row (record) represents one frame of LIN bus communication. First byte represents the command from MASTER and remains bytes represent a SLAVE answer from emulator's side. The enhanced checksum is defined by LIN bus standard as checksum including the data bytes and the identifier in addition and it is carried in the last (5th) byte of the frame. The EPB switch states are coded in the 3rd byte (Open, Close and Default). The checksum is calculated by slave node (EPB switch) e.g. by Arduino Micro's 8-bit AVR processor. We developed additional two security options. First is the implementation of so called "alive frame counter" (high nibble of 2nd byte in the frame), second option is calculation of additional checksum known as cyclic redundancy check byte (first byte in the frame). Both security options are defined in AUTOSAR standard. For CRC calculation was used the algorithm according AUTOSAR E2E Profile 1[14] and it is known as CRC-8-SAE J1850. Calculation of this checksum is also provided by AUTOSAR CRC library, which typically is quite efficient and may use hardware support. Useful educational information about CRC-8-SAE J1850 calculation and implementation details we found in [16]. The issue of functional safety for LIN bus-based ECU peripherals according automotive function safety standard ISO 26262 is beyond scope of this paper [15].

\section{CONCLUSION}

This article deals with analysis, design and implementation of the equipment that is designed for emulation of signals dedicated as inputs for electronic stability control ECU. We developed emulators of two key peripherals: one for wheel speed sensors (both two and three 
current levels types) and second for EPB switch. The main goal of our work was developing an educational system which is based on modern open-source technologies. This embedded system is based on the state of art embedded server-client software solutions with JavaScript. Besides of these educational aims we try to coexist with modern automotive trends - alignment of high level functional safety requirements together with using of peripherals which are equipped with car's buses. As illustration of these principles we proposed the EPB switch based on the LIN bus. Our possible contribution may be in the implementation of modern AUTOSAR network safety mechanism and parts of master/slave LIN bus state machines at open Arduino platform.

\section{References}

[1] REITZ, A. et all., "HARMONISATION OF THE RELEASE PROCESS FOR ELECTRIC PARKING BRAKE SYSTEMS," in Pub. FISITA, Proceedings of the EuroBrake 2016 Conference. 13-15 June 2016, Milan, Italy, 13-15 June 2016, MILAN, 2016.

[2] BOSCH, Encyclopedia of Automotive Engineering, John Wiley \& Sons, Ltd., 2014.

[3] BOSCH, "DF30H Basic Technical Documentation Sensor DF30H," 2015.

[4] PHILIPS SEMI., "KMI22/1 Rotational speed sensor for extended air gap application and direction detection," 2000.

[5] US Patent 20080105502, "'Electromechanical Parking Brake Device and Electronic System for Operating Same"," 2008. [Online]. Available: http://documents.allpatents.com/l/50722962/US20080105502A1. [Accessed 13. March 2013].

[6] BMW, ""E70 Voltage Supply and Bus Systems"," 2010. [Online]. Available: http://www.bmwmotorsports.org/pdf/e70/03a_E70\%20Voltage\%20Supply\%20and\%20Bus.pdf. [Accessed 13. March 2013].

[7] "AngularJS," [Online]. Available: https://angularjs.org/. [Accessed 13. March 2017].

[8] "Node.js - JavaScript runtime built on Chrome's V8 JavaScript engine," [Online]. Available: https://nodejs.org/en/. [Accessed 13. March 2017].

[9] "Arduino $\quad$ Micro," Anline]. https://www.arduino.cc/en/Main/arduinoBoardMicro. [Accessed 13. March 2017].

[10] "RASPBERRY PI 3 MODEL B," [Online]. Available: https://www.raspberrypi.org/products/raspberry-pi-3-model-b/. [Accessed 13. March 2017].

[11] "I2C interfacing Raspberry PI to Arduino," [Online]. Available: https://www.slideshare.net/MikeOchtman/i2c-interfacing-raspberry-pi-to-arduino. [Accessed 13. March 2017].

http://www.continental-

[12] CONTINENTAL, "Wheel Speed Sensor (WSS)," [Online]. Available: automotive.com/www/automotive_de_en/themes/commercial_vehicles/chassis_safety/speed_sensors/ wheel_speed_sensor_en.html. [Accessed 13. March 2017].

[13] MAXIM, "MAX9921 Dual, 2-Wire Hall-Effect Sensor Interface with Diagnostics," January 2010. [Online]. Available: https://www.maximintegrated.com/en/products/analog/sensorsand.../MAX9921.html. [Accessed 13. March 2017].

[14] AUTOSAR standard No.428 (release 4.2.2), "Specification of SW-C End-to-End Communication Protection Library," AUTOSAR, 2015.

[15] FURST,S., BMW, "AUTOSAR and Functional Safety," 8. November 2011. [Online]. Available: ttps://automotivetechis.files.wordpress.com/2013/04/autosar-and-functional-safety1.pdf. [Accessed 13. March 2017].

[16] MOLKENTHIN,B., "CRC Calculator (Javascript)," [Online]. Available: http://www.sunshine2k.de/coding/javascript/crc/crc_js.html. [Accessed 17 March 2017].

[17] US Patent 7540213, ""Gearshift lever"," 2009. [Online]. Available: http://www.freepatentsonline.com/7540213.html. [Accessed 13 March 2017]. 
Fig 1. EPB forecasted fitment EPB fitment rate [\%] [1] System [1]

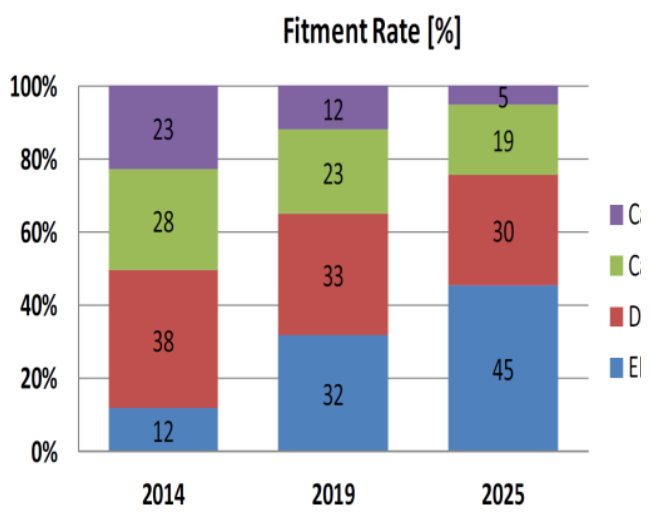

Fig.3 ECU type ESC with EPB functions configuration, adopted from [2]
Fig.2 Electric Parking Brake

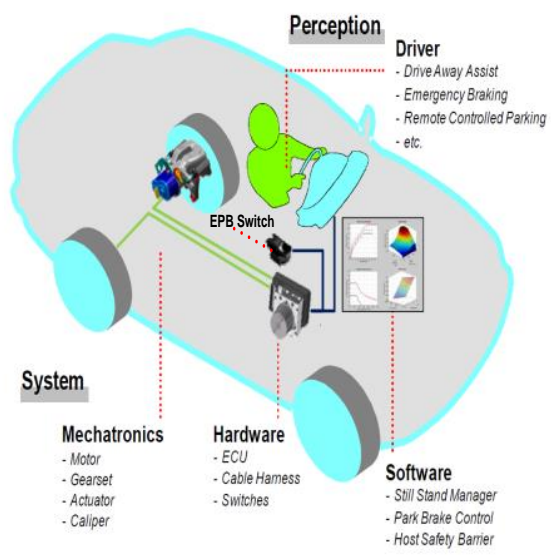

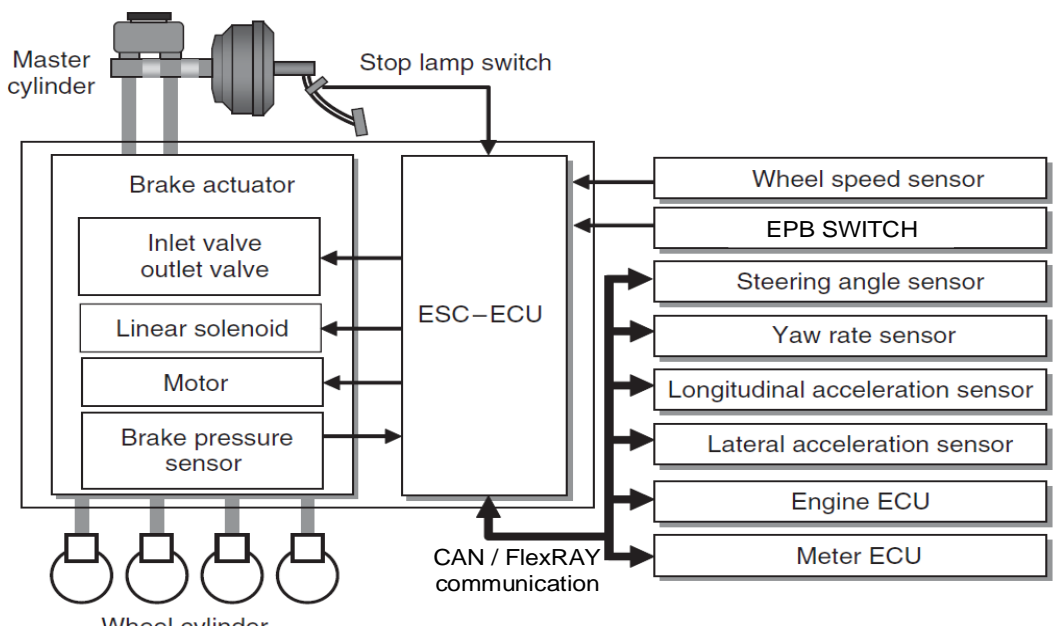

Fig.4 Three basic states of EPB electromechanical switch, adopted from [2] protocol type s, i, v [3]

Fig.5 Wheel speed sensors signal
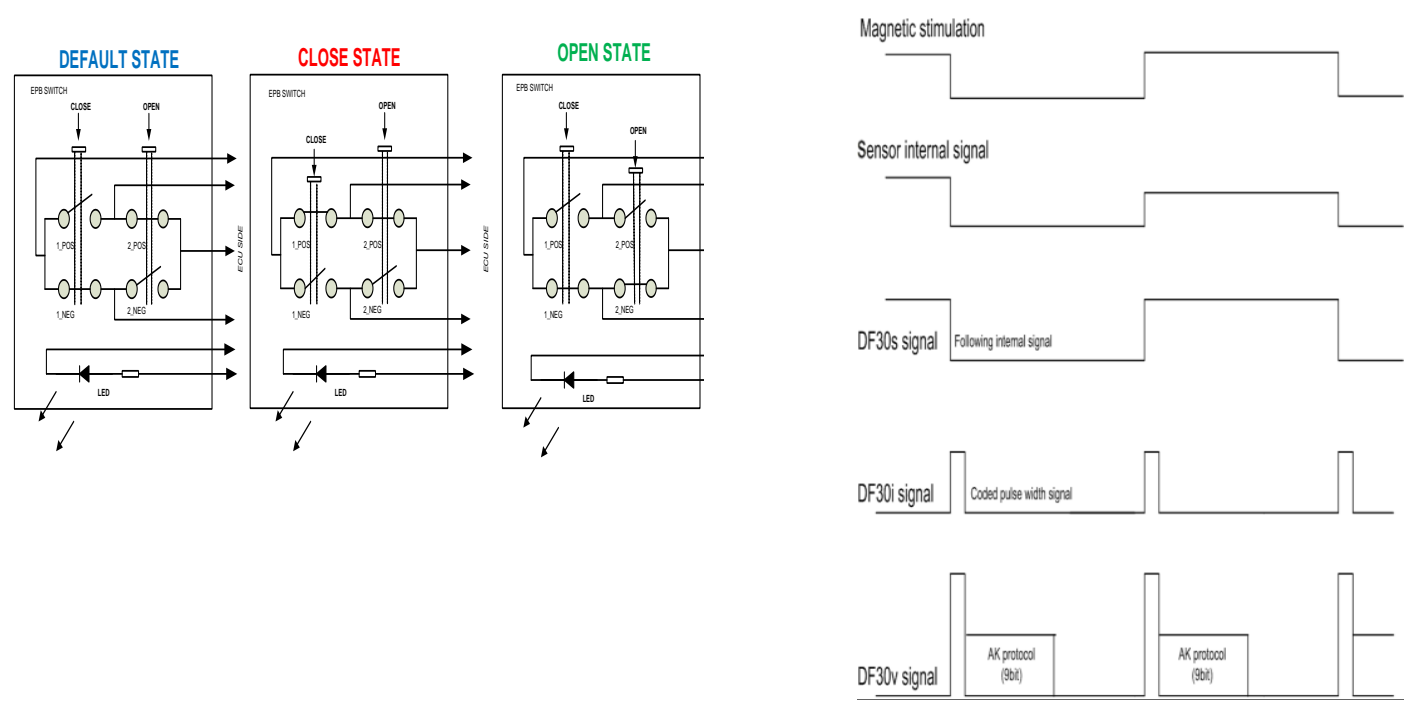
Fig.6 Emulation of the WSS DF11i - standstill idle current pulse (zero velocity)

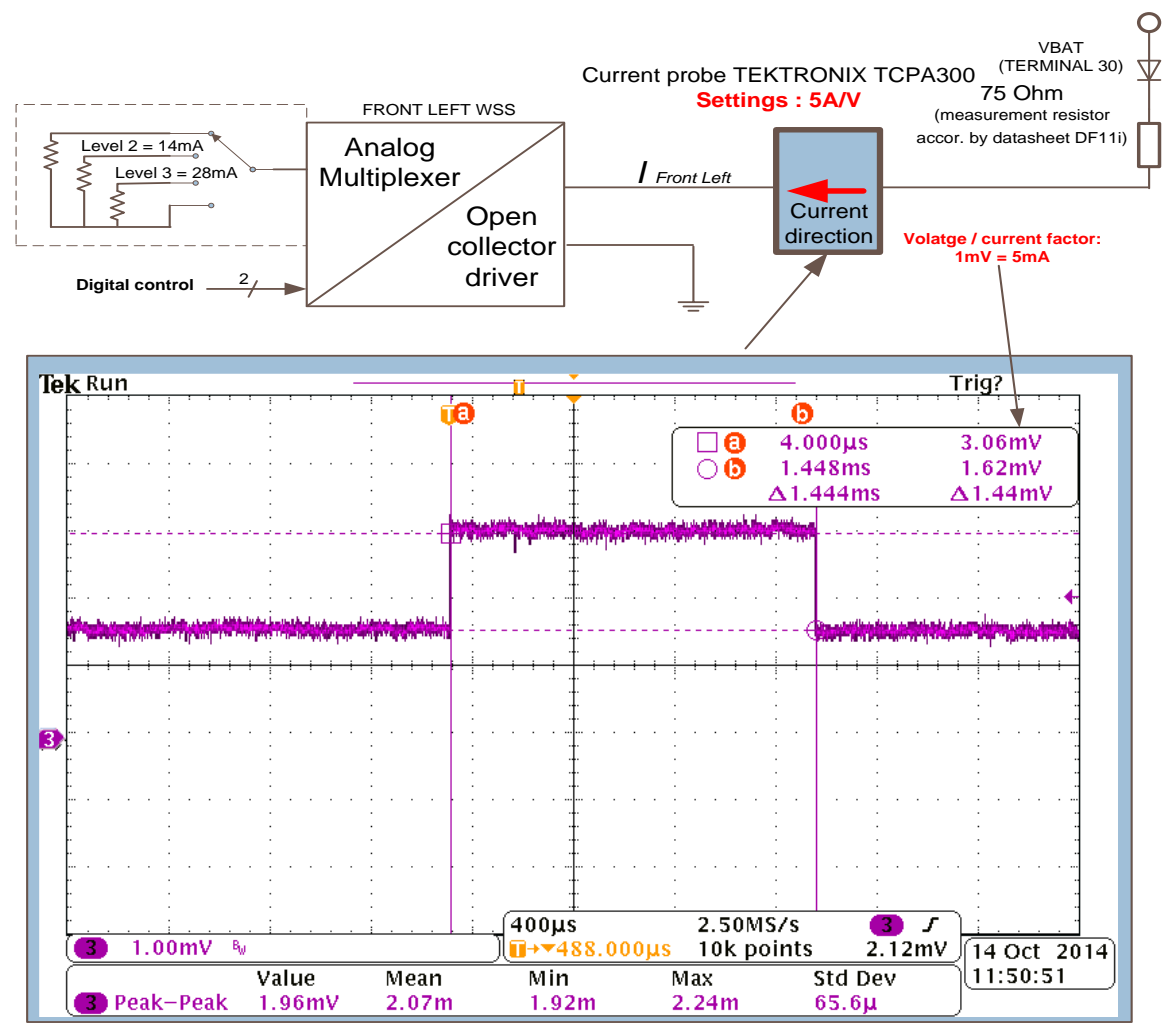

Fig.7 LIN bus communication frames between ECU side (MASTER) and LIN EPB switch emulator (SLAVE)

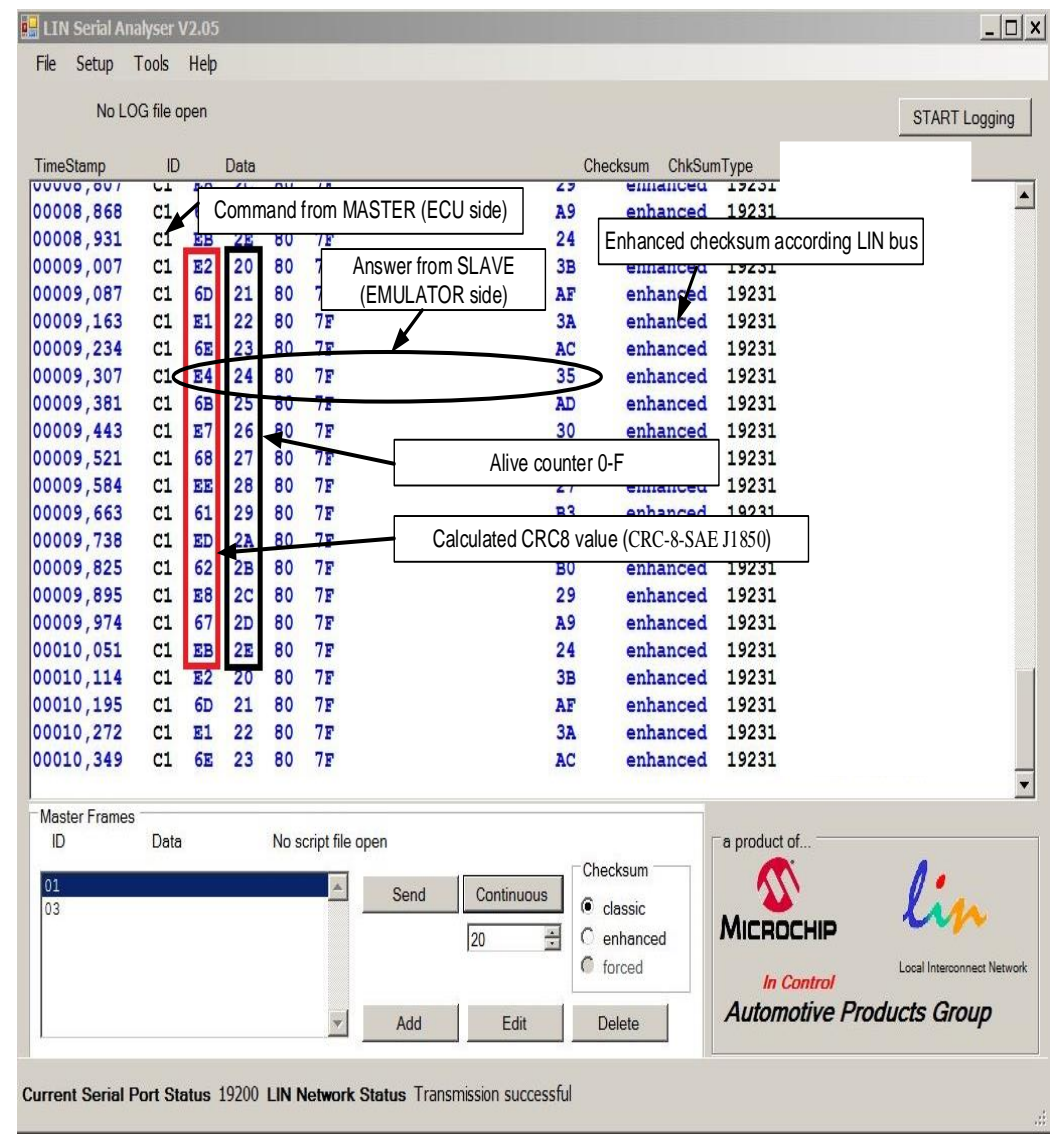


Fig.8 Blok diagram of the developed educational system for ECU peripheral's emulation

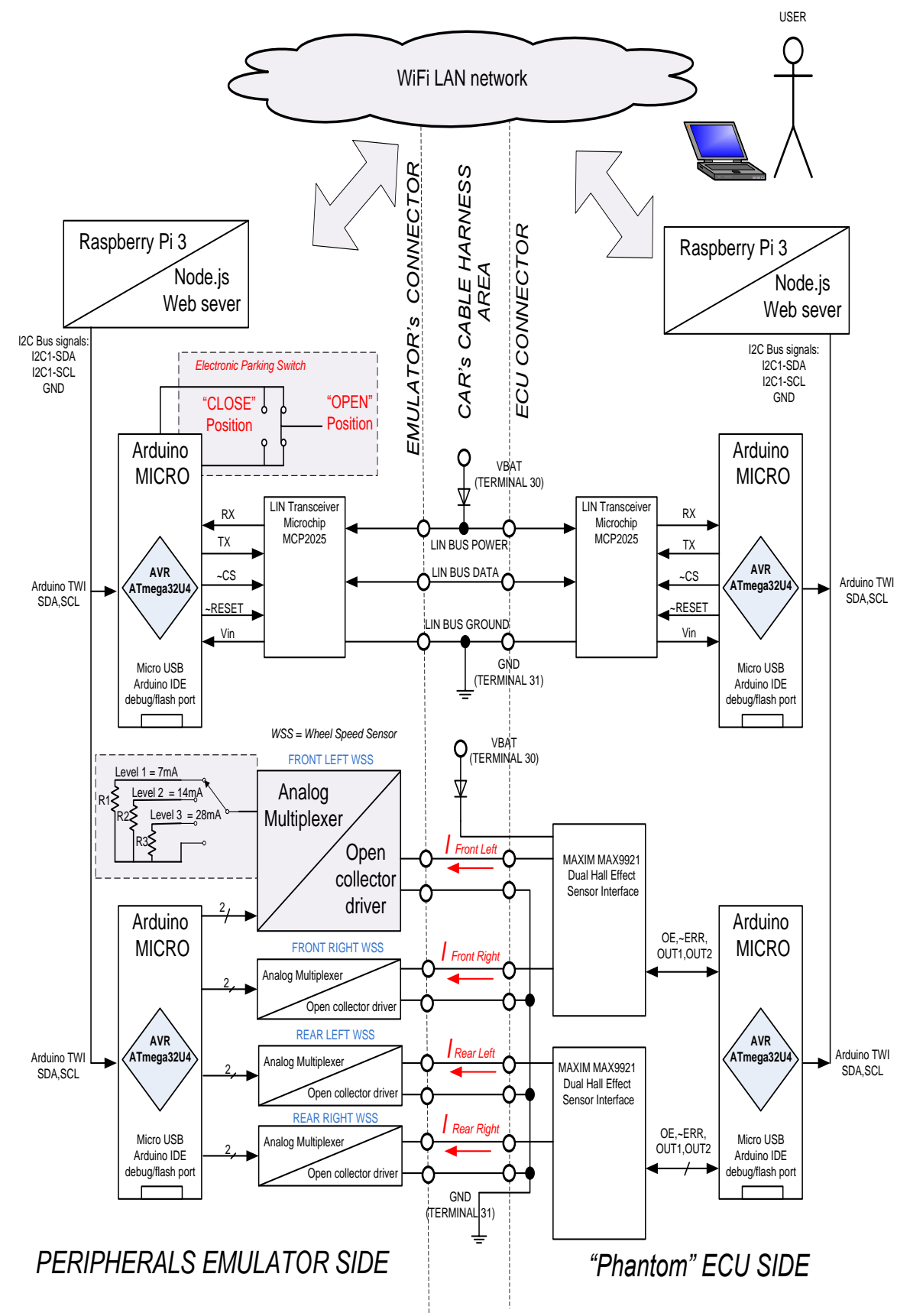

